\title{
On Tail Value-at-Risk for sums of non-independent random variables with a generalized Pareto distribution
}

\author{
Antonella Campana
}

Received: 10 August 2006/Revised: 24 May 2007/Published online: 8 October 2007

(C) The Geneva Association 2007

\begin{abstract}
Recently in actuarial literature several authors have derived lower and upper bounds in the sense of convex order for sums of random variables with given marginal distributions and unknown dependency structure. In this paper, we derive convex bounds for sums of non-independent and identically distributed random variables when marginal distributions are mixture models. In particular, we examine some well-known risk measures and we find approximations for Tail Value-at-Risk of the sums considered when marginal distributions are generalized Pareto distributions. By numerical examples we illustrate the goodness of the presented approximations.
\end{abstract}

Keywords Generalized Pareto distribution - Sums of random variables ·

Risk measures · Tail Value-at-Risk · Comonotonicity

JEL Classifications $\quad$ C16 $\cdot$ D81

\section{Introduction}

In financial or actuarial situations one often encounters random variables of the type $S=\sum_{i=1}^{m} X_{i}$ where the terms are not mutually independent and the multivariate distribution function of the random vector $\mathbf{X}=\left(X_{1}, X_{2}, \ldots, X_{m}\right)$ is not known. In many cases, only the marginal distribution functions of the random variables $X_{i}$ are specified.

\footnotetext{
A. Campana $(\bowtie)$

Dipartimento di Scienze Economiche Gestionali e Sociali, Università degli Studi del Molise, Via De Sanctis, Campobasso 86100, Italy

e-mail: campana@unimol.it

URL: www.unimol.it
} 
Recently in actuarial literature several authors have derived lower and upper bounds in the sense of convex order for sums of random variables when marginal distributions are given, but their joint distribution is unknown (Dhaene and Denuit 1999; Dhaene et al. 2002a; Kaas et al. 2000).

In this paper, we consider sums of non-independent and identically distributed random variables when marginal distributions are mixture models.

We derive convex bounds for such sums: upper bound is derived by using the concept of comonotonicity, lower bound is obtained by using the conditioning random variable $\Lambda$ in the mixture representation.

The convex bounds obtained can be used to evaluate risk measures for sums of non-independent random variables (Dhaene et al. 2003). We show how to find approximations for Tail Value-at-Risk when marginal distributions are generalized Pareto distributions. By numerical examples we illustrate the goodness of the presented approximations.

This paper is organized as follows. In Sect. 2, we present general results about convex bounds for sums of random variables. In Sect. 3, we introduce some wellknown risk measures for mixture models. In Sect. 4, we derive the generalized Pareto distribution as a mixture of models. In Sects. 5 and 6, we show how to evaluate the Tail Value-at-Risk for sums of non-independent and identically distributed random variables with a generalized Pareto distribution. The paper ends with numerical examples illustrating the goodness of the presented approximations.

\section{General results}

Let $\mathbf{X}=\left(X_{1}, X_{2}, \ldots, X_{m}\right)$ be a random vector possessing $F_{X_{1}}, F_{X_{2}}, \ldots, F_{X_{m}}$ as marginal cumulative distribution functions (cdf's, in short). Let $S$ be the sum of its components. In this paper, we restrict ourselves to non-negative random variables (rv's, in short) with finite expectations, further called risks.

For any random vector $\mathbf{X}=\left(X_{1}, X_{2}, \ldots, X_{m}\right)$ not necessarily comonotonic, its comonotonic counterpart is defined as any random vector with the same marginal distributions and with the comonotonic dependency structure. The concept of comonotonicity, its characterizations and applications in actuarial science and finance are examined in Dhaene et al. (2002a, 2002b). It can be proven that a random vector is comonotonic if and only if all its components are non-decreasing (or non-increasing) functions of the same rv.

The comonotonic counterpart of $\mathbf{X}$ will be denoted by $\mathbf{X}^{\mathbf{c}}=\left(X_{1}^{c}, X_{2}^{c}, \ldots, X_{m}^{c}\right)$.

Let $S^{c}$ be the sum of the components of $\mathbf{X}^{\mathbf{c}}$.

In Dhaene et al. (2002a), it is proved that the generalized inverse distribution of $S^{c}$, defined by

$$
F_{S^{c}}^{-1}(p)=\inf \left\{x \in \mathbf{R} \mid F_{S^{c}}(x) \geq p\right\}, \quad p \in(0,1),
$$

is given by the sum of the generalized inverse distributions of the marginal distributions: 


$$
F_{S^{c}}^{-1}(p)=\sum_{i=1}^{m} F_{X_{i}}^{-1}(p), \quad p \in(0,1) .
$$

In the sequel, we will refer to stop-loss order and convex order.

Definition 1 The $\mathrm{rv} X$ is said to precede the rv $Y$ in the stop-loss order sense $\left(X \leq_{s l}\right.$ $Y$ ) if and only if $X$ has lower stop-loss premiums than $Y$ :

$$
E\left[(X-d)_{+}\right] \leq E\left[(Y-d)_{+}\right] \quad d \in \mathbf{R} .
$$

Definition 2 The $\mathrm{rv} X$ is said to precede the rv $Y$ in the convex order sense $\left(X \leq_{c x}\right.$ $Y$ ) if and only if $X \leq_{s l} Y$ and in addition $E[X]=E[Y]$.

The following theorem (proved in Kaas et al. 2000) concerns convex order bounds for sums of dependent rv's.

Theorem 1 For any random vector $\mathbf{X}=\left(X_{1}, X_{2}, \ldots, X_{m}\right)$ and any $r v \Lambda$, we have that

$$
S^{l} \leq_{c x} S \leq{ }_{c x} S^{c}
$$

with $S^{l}$ given by

$$
S^{l}=\sum_{i=1}^{m} E\left[X_{i} \mid \Lambda\right]
$$

Differently of $\mathbf{X}^{\mathbf{c}}$, the random vector $\left(E\left[X_{1} \mid \Lambda\right], E\left[X_{2} \mid \Lambda\right], \ldots, E\left[X_{m} \mid \Lambda\right]\right)$ will in general not have the same marginal distributions of $\mathbf{X}$. If the conditioning $\operatorname{rv} \Lambda$ is such that all rv's $E\left[X_{i} \mid \Lambda\right]$ are non-increasing (or non-decreasing) functions of $\Lambda$, the lower bound $S^{l}$ is a sum of comonotonic rv's.

\section{Mixture models and risk measures}

Now we consider a rv $\Lambda$ with a continuous $\operatorname{cdf} F_{\Lambda}$.

We assume that, for any possible outcome $\Lambda$ of $\Lambda$, conditionally given $\Lambda=\lambda$, the risks $X_{i}$ are identically distributed and their conditional distribution $F_{X_{i} \mid \Lambda=\lambda}$ is such that all rv's $E\left[X_{i} \mid \Lambda\right]$ have a continuous cdf which depends on $\Lambda$.

The unconditional distribution of $X_{i}$ is the mixture distribution resulting from averaging their conditional distribution with respect to the mixing distribution $F_{\Lambda}$.

The concept of a mixture of distributions is very important in insurance, since insurance companies generally deal with heterogeneous risks. Mixture distributions are applied as models for claim numbers and insurance losses. For instance, the negative binomial distribution has been used successfully as a model for number of accidents for motorists (see Hogg and Klugman 1984): as it known, in this case the rv's $X_{i} \mid \Lambda=\lambda$ follow a Poisson distribution with mean $\lambda$ and the $\operatorname{rv} \Lambda$ has a gamma distribution. In the following sections, we'll study the Pareto case. 
In order to derive the distribution of the comonotonic upper bound $S^{c}$, we find that from (1), under our assumptions, it follows:

$$
S^{c} \stackrel{\mathrm{d}}{=} \sum_{i=1}^{m} F_{X_{i}}^{-1}(U) \stackrel{\mathrm{d}}{=} m X_{1},
$$

where the symbol $\stackrel{\mathrm{d}}{=}$ is used to indicate equality in distribution and $U$ is a rv that is uniformly distributed over the unit interval $(0,1)$.

For the lower bound $S^{l}$, we find:

$$
S^{l} \stackrel{\mathrm{d}}{=} m E\left[X_{1} \mid \Lambda\right] \stackrel{\mathrm{d}}{=} m h(\Lambda)
$$

where $h(\Lambda)$ is a continuous rv which is a function of $\Lambda$.

Now we consider some well-known risk measures like the Value-at-Risk (VaR), the Conditional Tail Expectation (CTE) and the Tail Value-at-Risk (TVaR) at level p.

For a rv $X$, for any $p$ in $(0,1)$, these risk measures are defined, respectively, by:

$$
\begin{gathered}
Q_{p}(X)=\inf \left\{x \in \mathcal{R} \mid F_{X}(x) \geq p\right\}=F_{X}^{-1}(p), \\
\operatorname{CTE}_{p}(X)=E\left[X \mid X>Q_{p}[X]\right], \\
\operatorname{TVaR}_{p}(X)=\frac{1}{1-p} \int_{p}^{1} Q_{q}(X) \mathrm{d} q
\end{gathered}
$$

If $F_{X}$ is continuous, then

$$
\operatorname{CTE}_{p}(X)=\operatorname{TVaR}_{p}(X) .
$$

Now let us consider the lower bound $S^{l}$ and the risk measures related to it. All the risk measures considered satisfy the property of positive homogeneity (Dhaene et al. 2003). Then, from (4) we find

$$
\begin{gathered}
Q_{p}\left(S^{l}\right)=m Q_{p}(h(\Lambda)), \\
\operatorname{CTE}_{p}\left(S^{l}\right)=m \operatorname{CTE}_{p}(h(\Lambda)), \\
\operatorname{TVaR}_{p}\left(S^{l}\right)=\operatorname{mTVaR}_{p}(h(\Lambda)) .
\end{gathered}
$$

The continuity of the distribution of the $\operatorname{rv} h(\Lambda)$ implies that

$$
C T E_{p}(h(\Lambda))=T \operatorname{VaR}_{p}(h(\Lambda))
$$

and

$$
\operatorname{CTE}_{p}\left(S^{l}\right)=\operatorname{TVaR}\left(S^{l}\right) .
$$

For instance, if the risks $X_{i}$ follow a negative binomial distribution, the risk measures related to the lower bound $S^{l}$ can be obtained replacing $h(\Lambda)$ by the $\operatorname{rv} \Lambda$ which is gamma distributed. 
For the risk measures related to the upper bound $S^{c}$, we find:

$$
\begin{aligned}
Q_{p}\left(S^{c}\right) & =m Q_{p}\left(X_{1}\right), \\
\operatorname{CTE}_{p}\left(S^{c}\right) & =m \operatorname{CTE}_{p}\left(X_{1}\right), \\
\operatorname{TVaR}_{p}\left(S^{c}\right) & =m \operatorname{TVaR}_{p}\left(X_{1}\right) .
\end{aligned}
$$

If the risks $X_{i}$ have a continuous distribution, the equality

$$
\operatorname{CTE}_{p}\left(S^{c}\right)=\operatorname{TVaR}_{p}\left(S^{c}\right)
$$

holds for any $p$ in $(0,1)$.

We know that Tail Value-at-Risk preserves stop-loss order, since it is a concave distortion risk measure (Dhaene et al. 2003). In our assumption, by Theorem 1, we have:

$$
\operatorname{TVaR}_{p}\left(S^{l}\right) \leq \operatorname{TVaR}_{p}(S) \leq \operatorname{TVaR}_{p}\left(S^{c}\right)
$$

Unfortunately, the quantiles (i.e., VaR) of $S^{l}, S$, and $S^{c}$ are not necessarily ordered in the same way.

From (18) it follows that the the range between $\operatorname{TVaR}_{p}\left(S^{l}\right)$ and $\operatorname{TVaR}_{p}\left(S^{c}\right)$ contains the exact and unknown $\operatorname{TVaR}_{p}(S)$.

\section{The generalized Pareto distribution}

Now we assume that the rv $\Lambda$ has a gamma distribution with parameters $\alpha$ and $\delta$ (and we write $\Lambda \sim G(\alpha, \delta)$ ). The probability density function (pdf, in short) of $\Lambda$ is:

$$
f_{\Lambda}(\lambda)=\frac{\delta^{\alpha}}{\Gamma(\alpha)} \lambda^{\alpha-1} \exp (-\delta \lambda), \quad \lambda>0 .
$$

Further, we assume that, for any possible outcome $\lambda$ of $\Lambda$, the conditional distribution of the risks $X_{i}$ is a gamma distribution with parameters $k$ and $\lambda$ (i.e., $\left.X_{i} \mid \Lambda=\lambda \sim G(k, \lambda)\right)$ :

$$
f_{X_{i} \mid \Lambda=\lambda}(x \mid \lambda)=\frac{\lambda^{k}}{\Gamma(k)} x^{k-1} \exp (-\lambda x), \quad x>0 .
$$

So we find that $E\left[X_{i} \mid \Lambda\right]=k \Lambda^{-1}$ and $\operatorname{Var}\left[X_{i} \mid \Lambda\right]=k \Lambda^{-2}$.

Of course, when $k=1$ the risks $X_{i}$ have a conditional exponential distribution.

We now develop, by compounding, a heavy-tailed skewed distribution (Hogg and Klugman 1984).

As it is known, the compound distributions are long and heavy-tailed distributions which have been used successfully as loss models by actuaries.

The weighting distribution for $\Lambda$ is the gamma distribution $G(\alpha, \delta)$. Thus the unconditional pdf of $X_{i}$ is: 


$$
\begin{aligned}
f_{X_{i}}(x) & =\int_{0}^{\infty} f_{X_{i} \mid \Lambda=\lambda}(x \mid \lambda) f_{\Lambda}(\lambda) \mathrm{d} \lambda \\
& =\int_{0}^{\infty} \frac{\delta^{\alpha} x^{k-1} \lambda^{\alpha+k-1} \exp (-(\delta+x) \lambda)}{\Gamma(\alpha) \Gamma(k)} \mathrm{d} \lambda .
\end{aligned}
$$

Comparing this integrand to the gamma pdf with parameters $\alpha+k$ and $\delta+x$, we see that:

$$
f_{X_{i}}(x)=\frac{\Gamma(\alpha+k)}{\Gamma(\alpha) \Gamma(k)} \frac{\delta^{\alpha} x^{k-1}}{(\delta+x)^{\alpha+k}}, \quad x>0 .
$$

which is the pdf of the generalized Pareto distribution (and we write $\left.X_{i} \sim G P(\alpha, \delta, k)\right)$.

When $k=1$, the mixture distribution is the Pareto distribution, a model often used to represent the loss distribution. While the cdf of the generalized Pareto distribution cannot be expressed in a simple closed form

$$
F_{G P(\alpha, \delta, k)}(x)=\frac{\Gamma(\alpha+k)}{\Gamma(\alpha) \Gamma(k)} \int_{0}^{x} \frac{\delta^{\alpha} t^{k-1}}{(\delta+t)^{\alpha+k}} \mathrm{~d} t, \quad x>0,
$$

the cdf of a Pareto distribution is:

$$
F_{X_{i}}(x)=1-\left(\frac{\delta}{\delta+x}\right)^{\alpha}, \quad x>0
$$

The mean and the variance of the generalized Pareto distribution are

$$
E\left[X_{i}\right]=\frac{\delta k}{\alpha-1},
$$

provided $\alpha>1$, and

$$
\operatorname{Var}\left[X_{i}\right]=\frac{\delta^{2} k(\alpha+k-1)}{(\alpha-1)^{2}(\alpha-2)}
$$

provided $\alpha>2$.

The generalized Pareto distribution plays an important role in extreme value theory. GPD fitting is one of the most useful concepts in the statistics of extremes: excesses over high thresholds can be modelled by a generalized Pareto distribution. The properties of this distribution are crucial for the statistical analysis of extremal events (Embrechts et al. 1997).

\section{Risk measures and convex bounds in the Pareto case}

Now we consider risk measures related to the sum $S$ of non-independent and identically distributed rv's with a generalized Pareto distribution. We assume that $\alpha>1$ to ensure a finite mean (see (24)). 
It is easy to prove that the comonotonic upper bound $S^{c}$ has a generalized Pareto distribution with parameters $\alpha, m \delta$ and $k$, i.e., $S^{c} \sim G P(\alpha, m \delta, k)$.

For the lower bound $S^{l}$, we find:

$$
S^{l} \stackrel{\mathrm{d}}{=} m k \Lambda^{-1}
$$

where the $\mathrm{rv} \Lambda^{-1}$ has a reciprocal gamma distribution. Its pdf is given by:

$$
f_{\Lambda^{-1}}(y)=f_{\Lambda}(1 / y) / y^{2}, \quad y>0 .
$$

Now let us consider risk measures related to the lower bound $S^{l}$. From (26), we find:

$$
\begin{aligned}
Q_{p}\left(S^{l}\right) & =m k Q_{p}\left(\Lambda^{-1}\right), \\
\operatorname{CTE}_{p}\left(S^{l}\right) & =m k C T E_{p}\left(\Lambda^{-1}\right), \\
\operatorname{TVaR}_{p}\left(S^{l}\right) & =m k T V a R_{p}\left(\Lambda^{-1}\right) .
\end{aligned}
$$

The continuity of the distribution of $\Lambda^{-1}$ implies that

$$
\operatorname{CTE}_{p}\left(\Lambda^{-1}\right)=\operatorname{TVaR}\left(\Lambda^{-1}\right)
$$

and

$$
\operatorname{CTE}_{p}\left(S^{l}\right)=\operatorname{TVaR}_{p}\left(S^{l}\right)
$$

It is straightforward to prove that the quantiles and conditional tail expectations of $\Lambda^{-1}$, for any $p$ in $(0,1)$, are given by:

$$
\begin{gathered}
Q_{p}\left(\Lambda^{-1}\right)=\frac{1}{F_{\Lambda}^{-1}(1-p ; \alpha, \delta)} \\
C T E_{p}\left(\Lambda^{-1}\right)=\frac{\delta}{(1-p)(\alpha-1)} F_{\Lambda}\left(F_{\Lambda}^{-1}(1-p ; \alpha, \delta) ; \alpha-1, \delta\right)
\end{gathered}
$$

where $F_{\Lambda}(\cdot ; \alpha, \delta)$ is the cdf of the gamma distribution with parameters $\alpha$ and $\delta$ and $F_{\Lambda}^{-1}(\cdot ; \alpha, \delta)$ its inverse function.

Therefore, we have:

$$
\operatorname{TVaR}_{p}\left(S^{l}\right)=\frac{m k \delta}{(1-p)(\alpha-1)} F_{\Lambda}\left(F_{\Lambda}^{-1}(1-p ; \alpha, \delta) ; \alpha-1, \delta\right) .
$$

In order to derive risk measures related to the upper bound $S^{c}$, let us introduce the incomplete beta function with parameters $k$ and $\alpha$ (which is available in many statistical software packages just like the gamma function and its inverse function):

$$
\beta(x ; k, \alpha)=\frac{\Gamma(\alpha+k)}{\Gamma(\alpha) \Gamma(k)} \int_{0}^{x} t^{k-1}(1-t)^{\alpha-1} \mathrm{~d} t, \quad 0<x<1 .
$$


By comparing (22) with (36), it is easy to show that:

$$
F_{G P(\alpha, \delta, k)}(x)=\beta\left(\frac{x}{\delta+x} ; k, \alpha\right), \quad x>0 .
$$

Let $\vartheta_{p}$ be the $p$-quantile of the beta distribution with parameters $k$ and $\alpha$, i.e.:

$$
\beta\left(\vartheta_{p} ; k, \alpha\right)=p .
$$

From (37) and (38) we derive:

$$
\vartheta_{p}=\frac{F_{G P(\alpha, \delta, k)}^{-1}(p)}{\delta+F_{G P(\alpha, \delta, k)}^{-1}(p)}
$$

with $F_{G P(\alpha, \delta, k)}^{-1}(p)=Q_{p}\left(X_{1}\right)$.

Then, from (3), we find that:

$$
\vartheta_{p}=\frac{Q_{p}\left(S^{c}\right)}{m \delta+Q_{p}\left(S^{c}\right)}
$$

Since $F_{G P(\alpha, \delta, k)}(\cdot)$ is continuous, we have:

$$
\operatorname{CTE}_{p}\left(S^{c}\right)=T \operatorname{VaR}_{p}\left(S^{c}\right) .
$$

Now we calculate $\operatorname{TVaR}_{p}\left(S^{c}\right)$ :

$$
\begin{aligned}
\operatorname{TVaR}_{p}\left(S^{c}\right) & =m \frac{\Gamma(\alpha+k)}{\Gamma(\alpha) \Gamma(k)} \int_{Q_{p}\left(X_{1}\right)}^{\infty} \frac{\delta^{\alpha} x^{k}}{(\delta+x)^{\alpha+k}} \mathrm{~d} x \\
& =\frac{m \delta k}{(1-p)(\alpha-1)}\left[1-F_{G P(\alpha-1, \delta, k+1)}\left(Q_{p}\left(X_{1}\right)\right)\right] \\
& =\frac{m \delta k}{(1-p)(\alpha-1)}\left[1-\beta\left(\vartheta_{p} ; k+1, \alpha-1\right)\right]
\end{aligned}
$$

Since

$$
\beta(x ; \alpha, k)=1-\beta(1-x ; k, \alpha), \quad 0<x<1,
$$

we obtain:

$$
\operatorname{TVaR}_{p}\left(S^{c}\right)=\frac{m \delta k}{(1-p)(\alpha-1)} \beta\left(1-\vartheta_{p} ; \alpha-1, k+1\right) .
$$

\section{Approximations for Tail Value-at-Risk for sums $S$}

If $T \operatorname{VaR}_{p}(S)$ is unknown, it can be approximated by risk measures related to the convex bounds $S^{l}$ and $S^{c}$ (see (18)). 
In Appendix, we graphically illustrate some numerical results obtained by computing the lower and upper bounds from (35) and (43).

We considered different values for the parameters $\alpha, \delta$, and $k$ of the generalized Pareto distribution. We set $m=1,000$.

By comparing numerical results corresponding to different values of $\alpha$, we observe that the smaller is $\alpha$, the better are the approximations obtained for $\operatorname{TVaR}_{p}(S)$.

The lower and upper bounds appear to be close to each other for small values of $\alpha$ (see Figs. 1 and 5). It is known that the parameter $\alpha$ controls how heavy a tail the distribution has: the smaller $\alpha$, the heavier the tail. So we find that the heavier is the distribution's tail, the smaller is the range between $\operatorname{TVaR}_{p}\left(S^{l}\right)$ and $\operatorname{TVaR}_{p}\left(S^{c}\right)$.

With regard to the parameters $\delta$ and $k$, they do not appear to have a significative impact on the numerical results.

We can conclude that the accuracy of the bounds obtained for $\operatorname{TVaR}_{p}(S)$ is high for a chosen set of parameter values where $\alpha$ is sufficiently small.

\section{Concluding remarks}

If the only information available concerning the multivariate distribution function of the random vector $\mathbf{X}$ consists of the marginal distribution functions of the $X_{i}, \operatorname{TVaR}_{p}(S)$ can be approximated by $\operatorname{TVaR}_{p}\left(S^{l}\right)$ or $\operatorname{TVaR}_{p}\left(S^{c}\right)$.

Replacing the cdf of $S$ by the cdf of $S^{c}$ is a prudent strategy in the framework of utility theory.

Replacing the cdf of $S$ by the cdf of $S^{l}$ gives an idea of the degree of overestimation of the risk involved by replacing $S$ by the less attractive rv $S^{c}$.

In this paper, we considered the generalized Pareto distribution, with parameters $\alpha, \delta$, and $k$, as marginal distribution.

By numerical illustrations we have seen that the accuracy of the bounds obtained for $\operatorname{TVaR}_{p}(S)$ is high for a chosen set of parameter values where $\alpha$ is sufficiently small.

These considerations can be extended to a generic concave distortion risk measure. In fact, it is known that a generic concave distortion risk measure can be approximated by a linear combination of Tail Value-at-Risk corresponding to different values $p$ (Dhaene et al. 2003).

Therefore, we can conclude that risk measures related to the convex bounds $S^{l}$ and $S^{c}$ provide good approximations to risk measures related to the sum $S$ of nonindependent and identically distributed rv's having a generalized Pareto distribution with a sufficiently small parameter $\alpha$ as marginal distribution.

Further research is needed to investigate other applications of the described approach and, in particular, to evaluate risk measures for sums of non-independent risks with discrete marginal distributions.

Acknowledgements The author is grateful to an anonymous referee for valuable comments. 


\section{Appendix}

The following figures display values of $\operatorname{TVaR}_{p}\left(S^{l}\right)$ and $T \operatorname{VaR} R_{p}\left(S^{c}\right)$ corresponding to some $p$, defined as in (35) and (43).

The parameters in Figs. 1, 4 and 5 are chosen to set the mean $E[S]$ constant and equal to 6,250 . We recall that $E[S]=E\left[S^{l}\right]=E\left[S^{c}\right]$.

Fig. $1 T \operatorname{TVaR}_{p}\left(S^{l}\right)$ and $\operatorname{TVaR}_{p}\left(S^{c}\right)$

Fig. $2 \operatorname{TVaR}_{p}\left(S^{l}\right)$ and $\operatorname{TVaR}_{p}\left(S^{c}\right)$
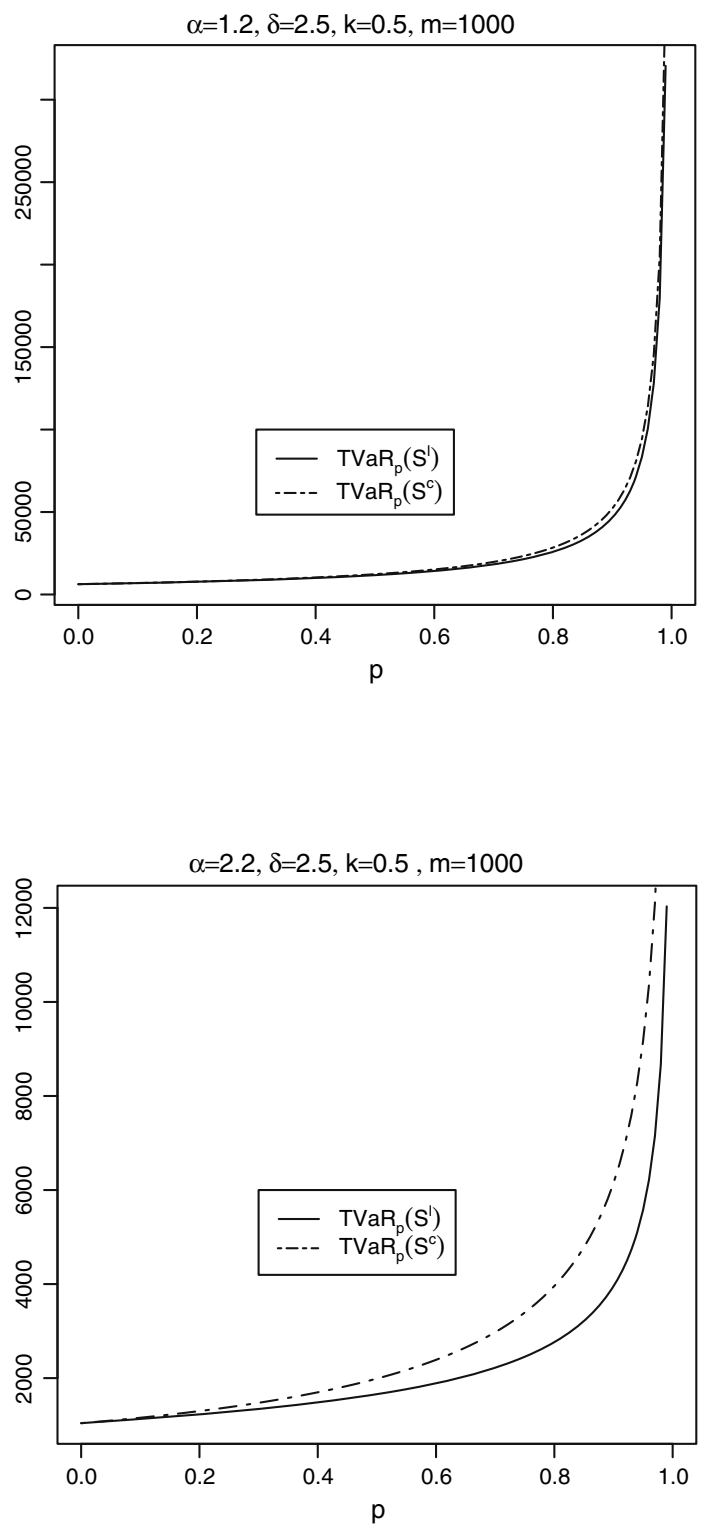


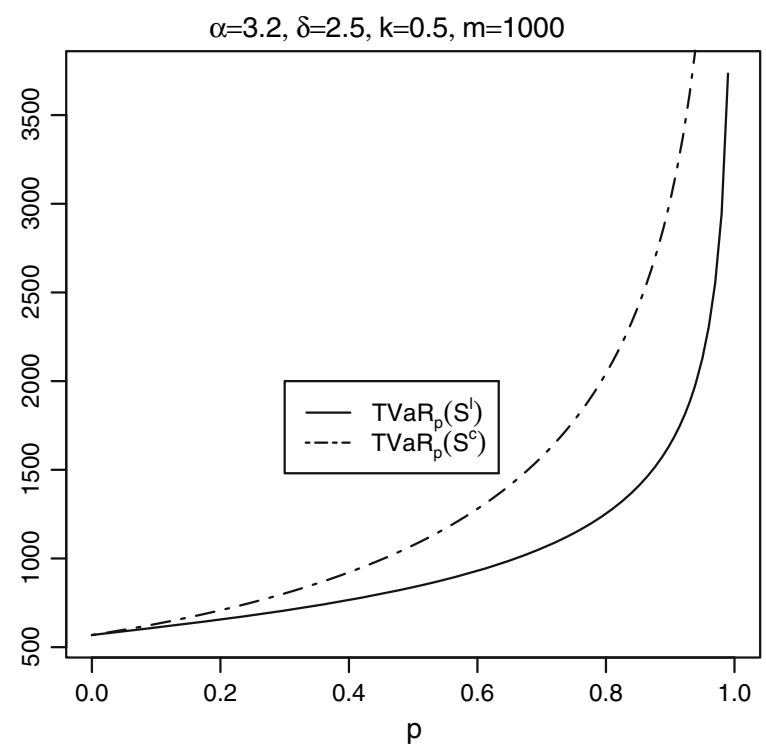

Fig. $3 \operatorname{TVaR}_{p}\left(S^{l}\right)$ and $\operatorname{TVaR}_{p}\left(S^{c}\right)$

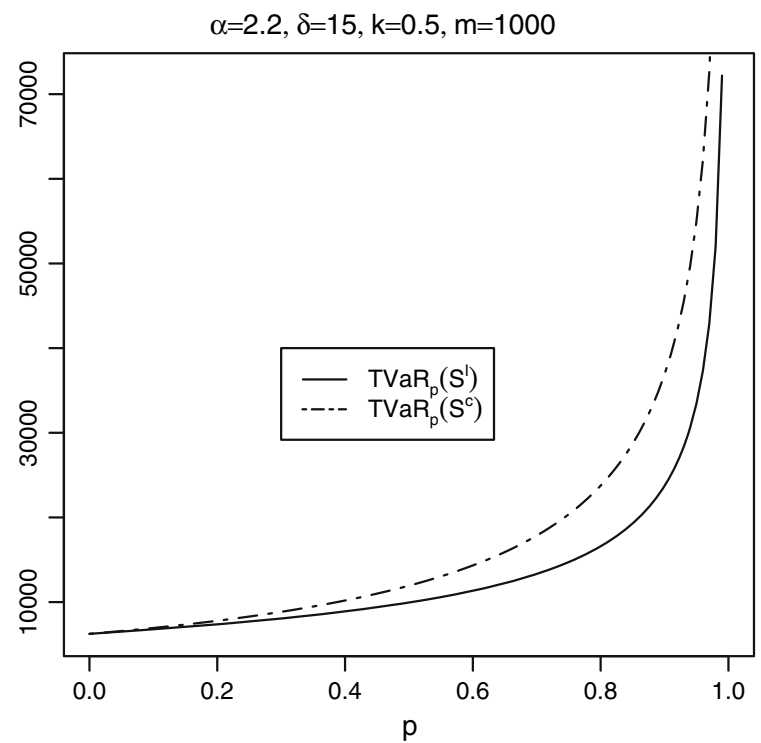

Fig. $4 \operatorname{TVaR}_{p}\left(S^{l}\right)$ and $\operatorname{TVaR}_{p}\left(S^{c}\right)$ 
Fig. $5 T \operatorname{TVaR}_{p}\left(S^{l}\right)$ and $\operatorname{TVaR}_{p}\left(S^{c}\right)$

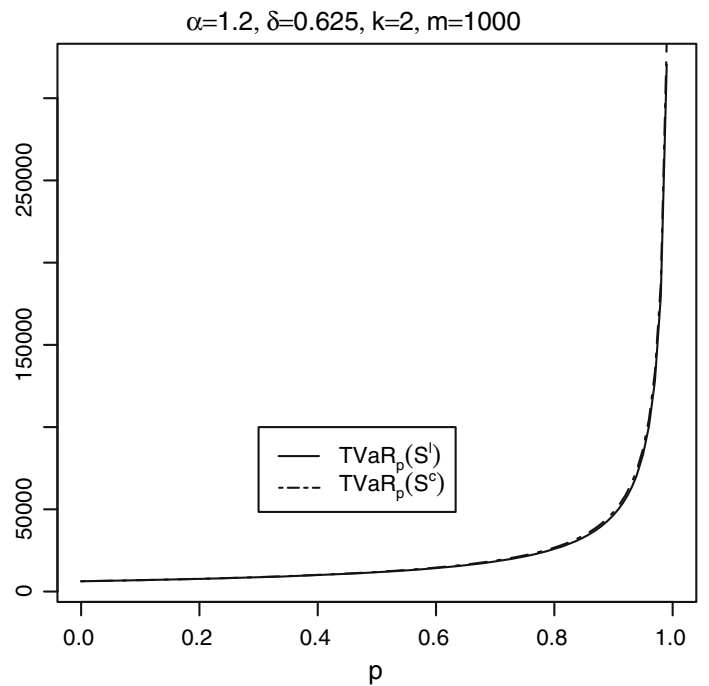

\section{References}

Dhaene J, Denuit M (1999) The safest dependence structure among risks. Insurance Math Econ 25:11-21 Dhaene J, Denuit M, Goovaerts MJ, Kaas R, Vynckce D (2002a) The concept of comonotonicity in actuarial science and finance: theory. Insurance Math Econ 31:3-33

Dhaene J, Denuit M, Goovaerts MJ, Kaas R, Vynckce D (2002b) The concept of comonotonicity in actuarial science and finance: applications. Insurance Math Econ 31:133-161

Dhaene J, Vanduffel S, Tang Q, Goovaerts MJ, Kaas R, Vynckce D (2003) Solvency capital, risk measures and comonotonicity: a review. Research Report OR 0416, Department of Applied Economics, K.U. Leuven

Embrechts P, Klüppelberg C, Mikosch T (1997) Modelling extremal events for insurance and finance. Springer-Verlag

Kaas R, Dhaene J, Goovaerts MJ (2000) Upper and lower bounds for sums of random variables. Insurance Math Econ 27:151-168

Hogg R, Klugman S (1984) Loss distributions. Wiley, New York 